\title{
Ivermectin and Zuranolone: A Double Standard in the Literature
}

\author{
Colin A. Ross* \\ Institute for Psychological Trauma, 1701 Gateway, Suite 349, Richardson, TX 75080, USA \\ *Corresponding author: Colin A. Ross, Institute for Psychological Trauma, 1701 Gateway, Suite 349, Richardson, TX 75080, USA
}

Received: February 21, 2022; Accepted: February 28, 2022; Published: March 10, 2022

Three recent publications illustrate the ongoing double standard concerning the effectiveness of ivermectin for treatment of early outpatient cases of COVID-19 infection. It seems to be true that ivermectin has no beneficial effect for seriously ill hospitalized patients - its potential utility is for reducing the frequency of transition from early mild outpatient cases to severely ill hospitalized cases. Lack of effectiveness inside hospitals does not prove a lack of effectiveness in outpatient populations.

\section{Zuranolone: A New Antidepressant}

A recent report by the manufacturers of zuranolone [1] concluded that, "Study supports the potential of zuranolone, when combined with standard of care, to accelerate the benefit of depression treatment compared to treatment with [antidepressants] alone." This conclusion was affirmed by an article in the American Psychiatric Association's Psychiatric Times [2]. The study involved 215 participants who received standard care with antidepressants and 210 for whom zuranolone was added to their standard care. Initial scores on the 17item Hamilton Rating Scale for Depression (HAM-D) were 26.8 (SD 2.5) for the zuranolone group and 26.2 (SD 2.6) for the standard care group. The score range for the HAM-D is 0-50 ( 8 items are scored 0-4 and 9 are scored $0-2$ ), and scores above 22 are regarded as indicating severe depression.

HAM-D score reductions at different time points in the 15-day study were:

\begin{tabular}{|l|c|c|c|}
\hline & Zuranolone Group & Standard Care Group & p value \\
\hline Day 3 & -8.9 & -7.0 & .0004 \\
\hline Day 8 & -11.3 & -9.2 & .0012 \\
\hline Day 12 & -12.8 & -11.4 & .0381 \\
\hline Day 15 & -13.7 & -12.9 & .2477 \\
\hline
\end{tabular}

At all points in the study the difference between the two groups on the HAM-D wasless than $4 \%$. This is a clinically meaningless difference, even though it was statistically significant at days 3,8 and 12 . By day 15 , the difference was no longer statistically significant. Nevertheless, the drug likely will be prescribed by many psychiatrists: the Psychiatric Times article stated that, "Study examining zuranolone treatment in patients with major depressive disorder (MDD) demonstrated a rapid and statistically significant reduction in depressive symptoms at day
3 and over the 2-week treatment period" [2]. The Psychiatric Times article skimmed over the fact that there was no difference between the groups at the end of the study. This is how things work in psychiatry: tiny effects of medications are hailed as advances in the field because they are statistically significant. Pharmaceutical companies sell many billions of dollars of psychiatric drugs per year, whereas ivermectin is a cheap generic medication.

\section{A Negative Study of Ivermectin}

When it comes to ivermectin, the situation is reversed. Highly clinically significant benefits are cited as evidence that ivermectin does not work for COVID-19 because the results were not statistically significant. In a recent study [3], ivermectin was added to standard care for 241 participants while standard care was provided to 249 participants. The authors concluded that, "The study findings do not support the use of ivermectin for patients with COVID-19." What were the results of the study? Mechanical ventilation was required in $1.7 \%$ of the ivermectin cases and $4.0 \%$ of the standard care cases (relative risk 0.41); ICU admissions were required in $2.4 \%$ of ivermectin cases and $3.2 \%$ of standard care cases (relative risk 0.78 ); and 28-day in-hospital deaths occurred in $1.2 \%$ of ivermectin cases and $4.0 \%$ of standard care cases (relative risk 0.31 ). Ivermectin reduced the frequency of 28 -day in-hospitals deaths by $69 \%$. The results of this study indicate that if ivermectin had been prescribed routinely to outpatients early in their course of infection, throughout the pandemic, hundreds of thousands of lives could have been saved worldwide.

\section{Concluding Thoughts}

Unscientific hostility toward ivermectin [4] as an outpatient treatment for COVID-19 continues in 2022. Physicians who recommend ivermectin are attacked, ostracized, fired from their jobs, canceled from social media, and threatened with board sanctions. Physicians who hail zuranolone as a significant step forward in the treatment of depression, on the other hand, get a round of applause from drug companies and their colleagues. They do not get accused of being anti-scientific, of being conspiracy theorists, or of spreading disinformation. It is unclear what financial or professional forces could ever change this pattern. 


\section{References}

1. Sage Therapeutics and Biogen announce the phase 3 CORAL Study met its primary and key secondary endpoints. BioSpace. News release. February 16, 2022. https:// www.biospace.com/article/sage-therapeutics-and-biogen-announce-the-phase-3coral-study-met-its-primary-and-key-secondary-endpoints/.

2. Kuntz L (2022) Improving depression symptoms: study meets endpoints. Psychiatric Times, February 16. https://www.psychiatrictimes.com/view/improving-depressionsymptoms-study-meets-endpoints.
3. Lim SC, Hor CP, Tay KH, Jelani AM, Tan WH, et al. (2022) Efficacy of ivermectin treatment on disease progression among adults with mild to moderate Covid-19 and comorbidities. The I-TECH randomized clinical trial. JAMA Internal Medicine doi:10.1001/jamainternmed.2022.0189.

4. Ross CA (2021) Thoughts on the Politics of COVID-19. Journal of Neurology and Neurocritical Care 4: 1-3. 